\title{
Mitochondrial pyruvate carrier 1 functions as a tumor suppressor and predicts the prognosis of human renal cell carcinoma
}

\author{
Xiao-Peng Tang ${ }^{1} \cdot$ Qian Chen ${ }^{2} \cdot \mathrm{Yi} \mathrm{Li}^{1} \cdot$ Yan Wang ${ }^{2} \cdot \mathrm{Hong}^{-B o} \mathrm{Zou}^{2} \cdot$ Wen-Juan $\mathrm{Fu}^{2} \cdot \mathrm{Qin} \mathrm{Niu}^{2} \cdot \mathrm{Qian}-\mathrm{Guang} \mathrm{Pan}^{1} \cdot$ \\ Pei Jiang ${ }^{1} \cdot$ Xiao-Song $\mathrm{Xu}^{1} \cdot \mathrm{Ke}-\mathrm{Qin} \mathrm{Zhang}{ }^{1} \cdot$ Hong $\mathrm{Liu}^{1} \cdot \mathrm{Xiu}-\mathrm{Wu}$ Bian $\mathbb{D}^{2} \cdot$ Xiong-Fei $\mathrm{Wu}^{1}$
}

Received: 11 March 2018 / Revised: 1 August 2018 / Accepted: 20 August 2018 / Published online: 5 October 2018

(c) United States \& Canadian Academy of Pathology 2018

\begin{abstract}
Invasion and subsequent metastasis are major characteristics of malignant human renal cell carcinoma (RCC), though the mechanisms remain elusive. Mitochondrial pyruvate carrier (MPC), a key factor that controls pyruvate transportation in mitochondria, is frequently dysregulated in tumor cells and loss of MPC predicts poor prognosis in various types of cancer. However, the clinical relevance and functional significance of MPC in RCC remain to be elucidated. In this study, we investigated the expression of MPC1 and MPC2 in specimens from RCC patients and observed downregulation of MPC1, but not MPC2, in RCC tissues compared with adjacent non-cancerous tissue. Moreover, RCC patients with higher MPC1 expression exhibited longer overall survival rate than those with lower MPC1. Functionally, MPC1 suppressed the invasion of RCC cells in vitro and reduced the growth of RCC cells in vivo, possibly through inhibition of MMP7 and MMP9. Further studies revealed that loss of MPC1 was induced by hypoxia in RCC cells, and notably, MPC1 expression, was negatively correlated with HIF1 $\alpha$ expression in RCC cells and patient samples. Taken together, our results identify antitumor function of MPC1 in RCC and revealed MPC1 as a novel prognostic biomarker to predict better patient survival.
\end{abstract}

\section{Introduction}

Renal cell carcinoma (RCC) represents $90-95 \%$ of kidney cancer. Although the mortality rate of RCC has been decreasing due to nephrectomy, immunotherapy and targeted therapy, invasion and subsequent metastasis still occur in $25-30 \%$ patients and lead to death [1]. Therefore, clarifying the mechanisms that control the

These authors contributed equally: Xiao-Peng Tang, Qian Chen.

Electronic supplementary material The online version of this article (https://doi.org/10.1038/s41374-018-0138-0) contains supplementary material, which is available to authorized users.

Xiu-Wu Bian

bianxiuwu@263.net

Xiong-Fei Wu

wuxiongfei18@hotmail.com

1 Department of Nephrology, Southwest Hospital, Third Military Medical University (Army Medical University), 400038 Chongqing, China

2 Institute of Pathology, Southwest Hospital, Third Military Medical University (Army Medical University), 400038 Chongqing, China invasion of RCC is critical for the development of novel therapeutic strategies.

Accumulating evidence reveals pivotal roles of aberrant mitochondrial metabolism in tumorigenesis [2]. Key molecules associated with mitochondrial metabolism are tightly involved in tumor progression [3]. Mitochondrial pyruvate carrier (MPC), including two members, MPC1 and MPC2, was originally described as gatekeepers responsible for pyruvate transportation and oxidation in mitochondria [4]. Cancer-related functions of MPC have also been identified in several types of cancer. For example, depletion of MPC or blocking its activity by specific inhibitor results in aerobic glycolysis and aggressive growth of tumor cells [2]. Decreased expression of MPC protein is correlated with poor survival of patients with various types of cancer, including colon cancer [5], prostate cancer [6, 7], and esophageal cancer [8]. Overexpression of MPC in colorectal cancer cell lines decreased the tumorigenicity of subcutaneously inoculated tumor cells in mice [5]. Moreover, in colon cancer and esophageal cancer [5, 8], MPC suppresses the Warburg effect, which is one of the most important features in cancer cells. Interestingly, recent work by Ohashi et al. showed that MPC1 is an indicator for favorable prognosis of intrahepatic cholangiocarcinoma and inhibits tumor 
invasion and metastasis [9]. These results suggest that MPC may function as a tumor suppressor through regulation of metabolism and epithelial-mesenchymal transition (EMT) of tumor cells.

Despite the potential involvement of MPC in cancer invasion and metastasis, the clinical relevance and functional mechanism of MPC in RCC remain to be elucidated. In this study, we investigated the expression of MPC in human RCC and adjacent non-cancerous tissue. Our data suggest that the expression of MPC1, but not MPC2, was downregulated in RCC tissue compared with paired noncancerous tissue, and the expression levels were negatively associated with tumor size, advanced lymph node metastasis, and overall survival (OS) of patients. Additionally, knockdown of MPC1 increased the migration and invasion of RCC cells. In contrast, overexpression of MPC1 in human RCC cells inhibited their migration, invasion, and tumorigenesis in xenograft models. Mechanistically, we observed that MPC1 expression impaired the mobility of RCC cells potentially through inhibiting hypoxia inducible factor $1 \alpha$ subunit $(\mathrm{HIF} 1 \alpha)$. Thus, our data imply that MPC1 may control the progression of RCC and acts as a novel prognostic indicator for human RCC.

\section{Materials and methods}

\section{Patients and tissue specimens}

Twelve pairs of tumor and adjacent non-cancerous tissues from RCC patients who underwent surgical resection in the Southwest Hospital, Third Military Medical University (Army Medical University) from January 2014 to December 2016, Chongqing, China with approval from the Institutional Ethics Committee were examined by Western blotting. Two tissue microarrays including $150 \mathrm{RCC}$ tissues without radiotherapy or chemotherapy before surgery were purchased from BioChip (Shanghai, China) (Supplementary Table 1), in which 30 RCC cases included. All patients were followed up after operation. All patients were provided with informed consent. An independent cohort of 164 patient specimens from The Cancer Genome Atlas (TCGA) database was reviewed to validate the prognostic value of MPC1 in RCC.

\section{Immunohistochemical staining}

Human tissue sections were deparaffinized and hydrated by a series of xylene and alcohol treatment. The sections were incubated with rabbit polyclonal anti-MPC1 (Abcam, Cambridge, UK), and mouse polyclonal anti-HIF1 $\alpha$ (Abcam) antibodies at $4{ }^{\circ} \mathrm{C}$ overnight, followed by incubation with avidin-biotin-peroxidase (DAKO). MPC1 and
HIF $1 \alpha$ were considered positive by cytoplasmic staining and the expression levels were semi-quantitatively analyzed using a composite score system by assessing both the percentage and intensity of stained tumor cells. The percentage of positive cells was calculated in high-power fields (HPF) as follows: [10] sections with $<1 \%$ positive cells were rated as $0 ; 1-25 \%$ positive cells as $1 ; 26-50 \%$ positive cells as 2 ; $51-75 \%$ positive cells as 3 , and $76-100 \%$ positive cells as 4 . The staining intensity was rated as follows: 1 for weak intensity; 2 for moderate intensity; and 3 for high intensity. Points for staining intensity and the percentage of positive cells were multiplied. Tumor specimens were classified into three groups according to overall scoring: $0-1$ as negative expression, 2-4 as weak expression, and 6-12 as high expression. Total scores were expressed as: $0-4$ as low and 6-12 as high. All sections were evaluated independently by two pathologists without knowledge of the identity of patients and the clinical outcome.

\section{Reagents}

UK5099, a MPC inhibitor, and $\mathrm{CoCl}_{2}$, which elicits a hypoxia condition in cell culture, were from Sigma-Aldrich (St. Louis, MO).

\section{Cell culture}

The 786-O RCC cell line was obtained from ATCC and maintained in DMEM supplemented with $10 \%$ fetal bovine serum (FBS) (Invitrogen, Carlsbad, CA, USA).

\section{Lentiviral infection}

For overexpression of MPC1-Flag in RCC cell line 786-O, full length human MPC1-Flag was generated and inserted into a lentivirus vector. The 786-O cell line $\left(1 \times 10^{6} /\right.$ well $)$ was infected with lentivirus containing the constructs. The cells were selected by $4 \mu \mathrm{g} / \mathrm{ml}$ puromycin (Solarbio Science $\&$ Technology, Beijing, China). $786-\mathrm{O}$ cells $\left(1 \times 10^{6} /\right.$ well $)$ were also infected with siMPCl or empty control lentivirus vectors (siCtrl) (Genechem, Shanghai, China). The sequences for the siRNAs were: MPC1-RNAi-1 5'UCCCTCTUTTUCTATTCTTTU-3', and MPC1-RNAi-2 5'-UUCTTATCAAACACUAUATUA-3'.

\section{Tumor cell invasion and migration assay}

Tumor cells in DMEM basic medium $\left(2 \times 10^{4} /\right.$ well $)$ were plated in the upper transwells. The lower transwells were filled with DMEM medium containing 10\% FBS as cell migration inducer. After $24 \mathrm{~h}$ incubation, non-migrating cells were removed from the upper surface of the membranes, and the cells on the lower surface of the membranes 

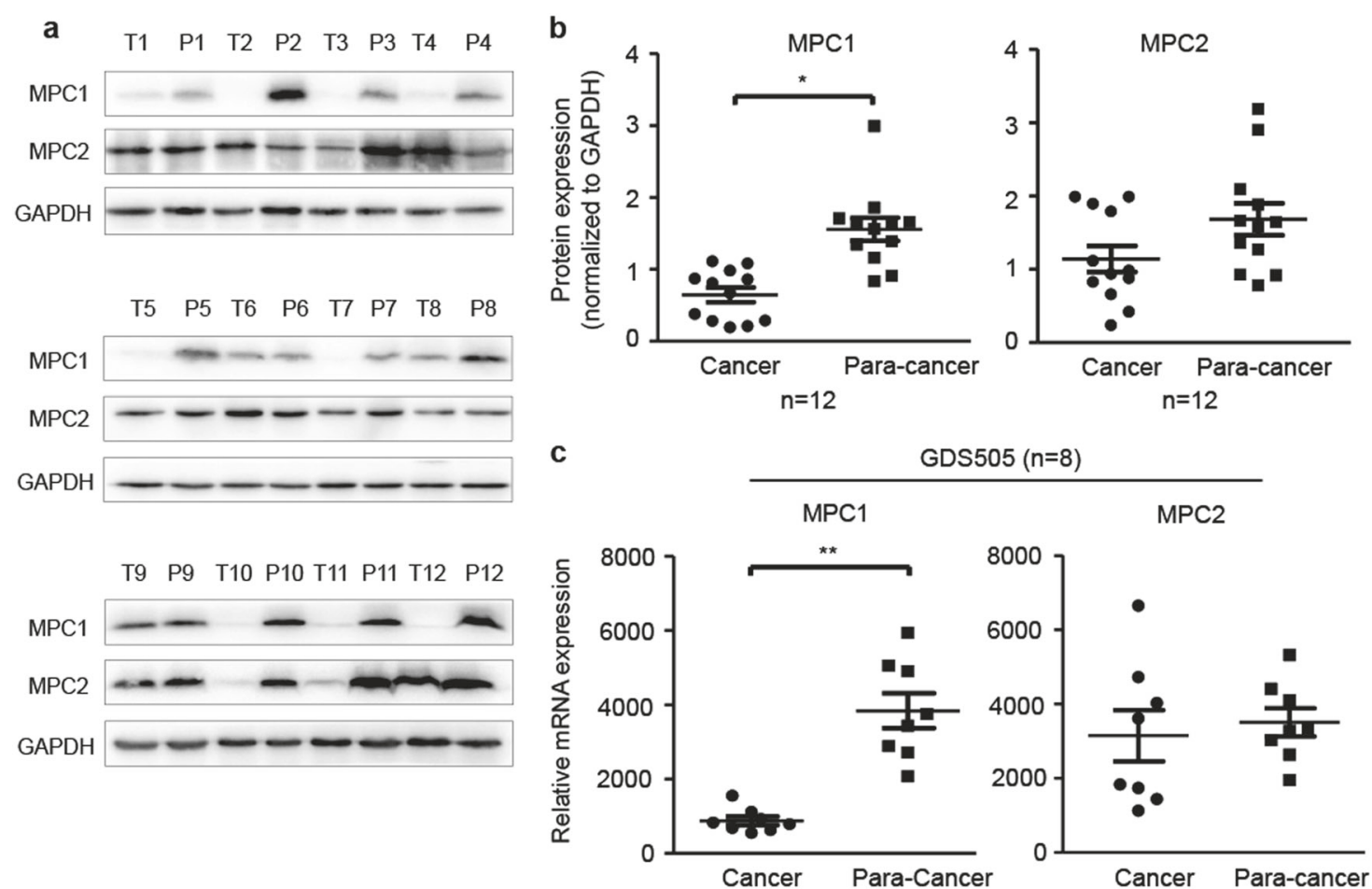

d
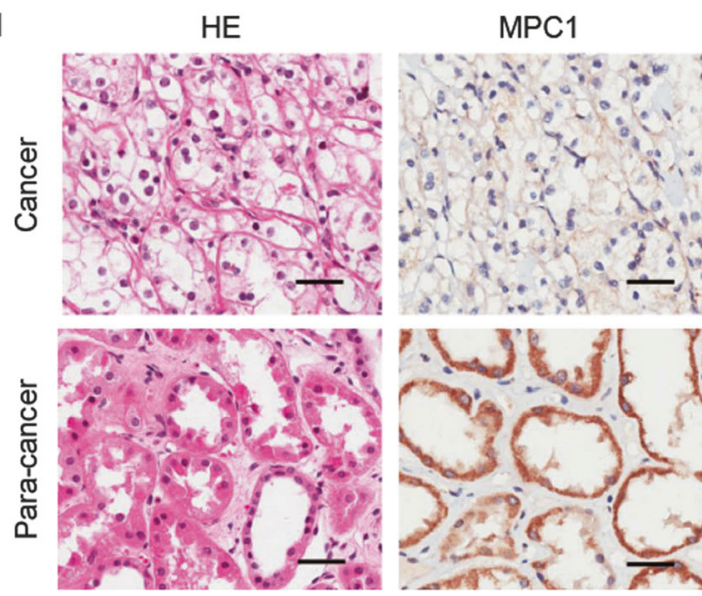

e

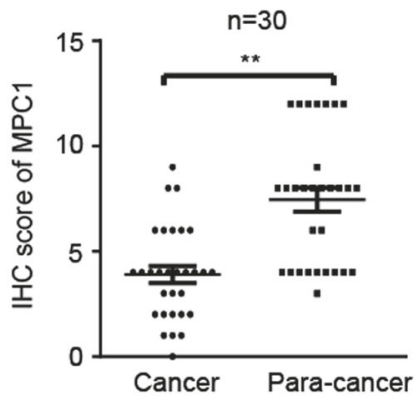

Fig. 1 The expression of MPC1 protein and mRNA in human RCC and adjacent non-cancerous tissue. a Western blotting of MPC1 and MPC2 in 12 paired RCC (T) and adjacent non-cancerous tissue (P). GAPDH serves as a loading control. b Quantitative analysis of MPC1 and MPC2 expression in 12 paired RCC and adjacent non-cancerous tissue, $* P<0.05$. c Analyses of MPC1 and MPC2 mRNA levels in eight paired RCC tissues from GDS505 database, $* * P<0.01$. d Representative immunohistochemistry (IHC) staining for MPC1 (brown color) in RCC and adajacent non-cancerous tissue. Scale bar $=$ $50 \mu \mathrm{m}$. e IHC score of MPC1 in tumor and adjacent non-cancerous tissue in 30 paired RCC specimens, $* * P<0.01$ were stained with crystal violet (Beyotime, Jiangsu, China). For invasion assay, BD Matrigel ${ }^{\mathrm{TM}}$ (BD Biosciences, San Jose, CA, USA) was added into transwell chambers before the cells were seeded. The invading and migrating cells in at least five randomly selected microscopic fields at $\times 200$ magnification were counted under light microscopy. The data was expressed as the mean $\pm \mathrm{SE}$ of cells per field in triplicate.

\section{Western blotting}

Western blotting was performed as previously described [11]. Primary antibodies used were: rabbit polyclonal anti-MPC1 (Cell Signaling, Beverly, MA), rabbit polyclonal anti-MPC2 (Abcam), mouse polyclonal anti-HIF1 $\alpha$ (Abcam), rabbit polyclonal anti-MMP7 (Cell Signaling), rabbit polyclonal anti-MMP9 (Cell 
Fig. 2 The correlation between the expression levels of MPC1 and patient prognosis. a Representative IHC staining for MPC1 in RCC specimens with different TNM stages (I, II, and III). Scale bar $=10 \mu \mathrm{m}$. b The correlation between IHC scores of MPC1 expression in RCC tumors with different TNM stages (I, II, and III). $* P<0.05$; $* * P<0.01$. c Kaplan-Meier analysis of overall survival rate (OS) in 150 RCC patients with MPC $1^{\text {low }}$ and MPC $1^{\text {high }}$ RCC. d Kaplan-Meier analyses of OS of 164 RCC patients from TCGA database a
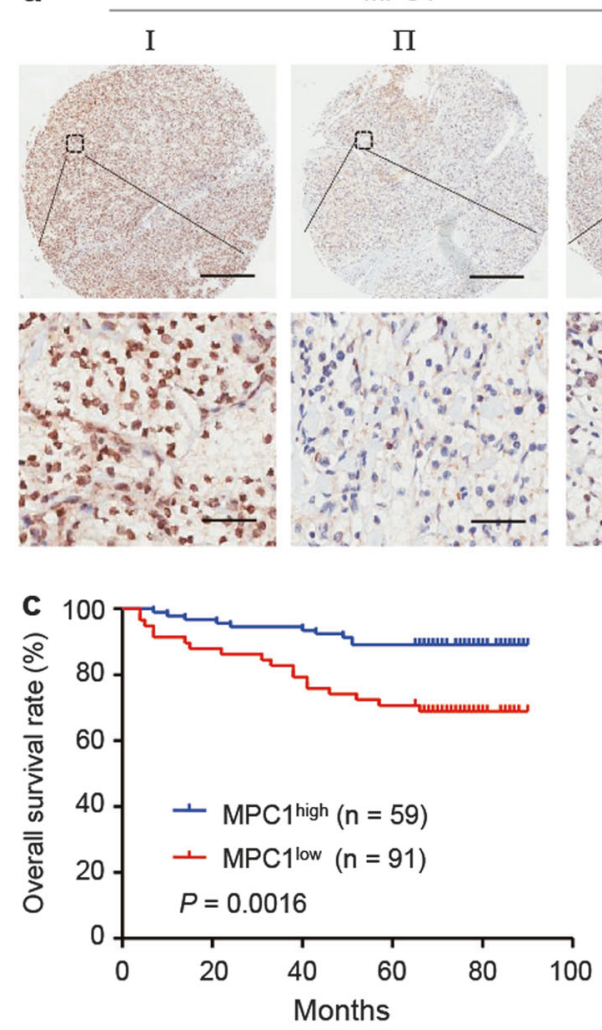

b

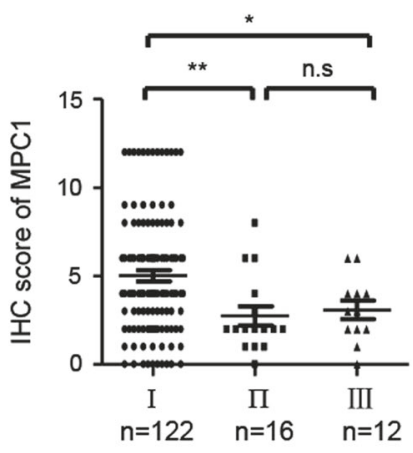

Signaling), and rabbit polyclonal anti-GAPDH (Cell Signaling) antibodies.

\section{qRT-PCR}

Total RNA was extracted from tumor cells with RNAiso reagent (Takara, Dalian, China). Reverse-transcription and quantitative Real-Time PCR were performed by using One Step SYBR Prime Script RT-PCR kit (Takara). Specific primers for amplification were listed in Supplementary Table 2. All experiments were performed in quadruplicate samples with results plotted as the mean \pm SD.

\section{Xenografts}

All animal experiments were approved by the Institutional Animal Care and Use Committee of the Southwest Hospital, Third Military Medical University (Army Medical University). A total of $1 \times 10^{5}$ MPC1overexpressing or control cells were suspended in $50 \mu \mathrm{l}$ PBS and subcutaneously injected into 6-week-old female NOD-SCID mice (Laboratory Animal Center, Southwest Hospital, Third Military Medical University, China). The size of resultant tumors was measured every 7 days for a month using a Vernier caliper and tumor volume was calculated as: shortest diameter ${ }^{2} \times$ longest diameter $/ 2$. The animals were sacrificed 30 days after tumor cell implantation and subcutaneous xenograft tumors were analyzed by IHC. Tumor tissue sections deparaffinized and hydrated by a series of xylene and alcohol treatment were incubated with rabbit polyclonal anti-MPC1 (Abcam), rabbit polyclonal anti-MMP7 (Cell signaling), and mouse polyclonal anti-HIF1 $\alpha$ (Abcam) antibodies at $4{ }^{\circ} \mathrm{C}$ overnight, followed by incubation with avidin-biotin-peroxidase (DAKO).

\section{Statistical analysis}

All experiments were performed at least three times with triplicate samples. Statistical analyses were conducted using the SPSS software 18.0. The correlation between MPC1 expression and the clinicopathological features of RCC patients was accessed by the Pearson chisquared $\chi^{2}$ test. Survival curves were calculated by the Kaplan-Meier method, and comparisons were made by the log-rank test. Paired Student's $t$-test for two groups and one-way ANOVA for multiple groups were used. The cutoff value of IHC score was determined by relative risk analysis with the statistical software X-tile [12]. 
Fig. 3 The mobility of RCC cells with MPC1 deficiency. a Migration and invasion analyzed by transwell assays in 786-O cells transfected with siCtrl and siMPC1, $* P<0.05$. b The motility of 786-O cells with or without the treatment of MPC inhibitor UK5099, $* P<0.05$. c The invasion and migration of 786-O cells with stable transfection of control vector (Mock) or MPC1 (OE-MPC1), $* P<0.05$
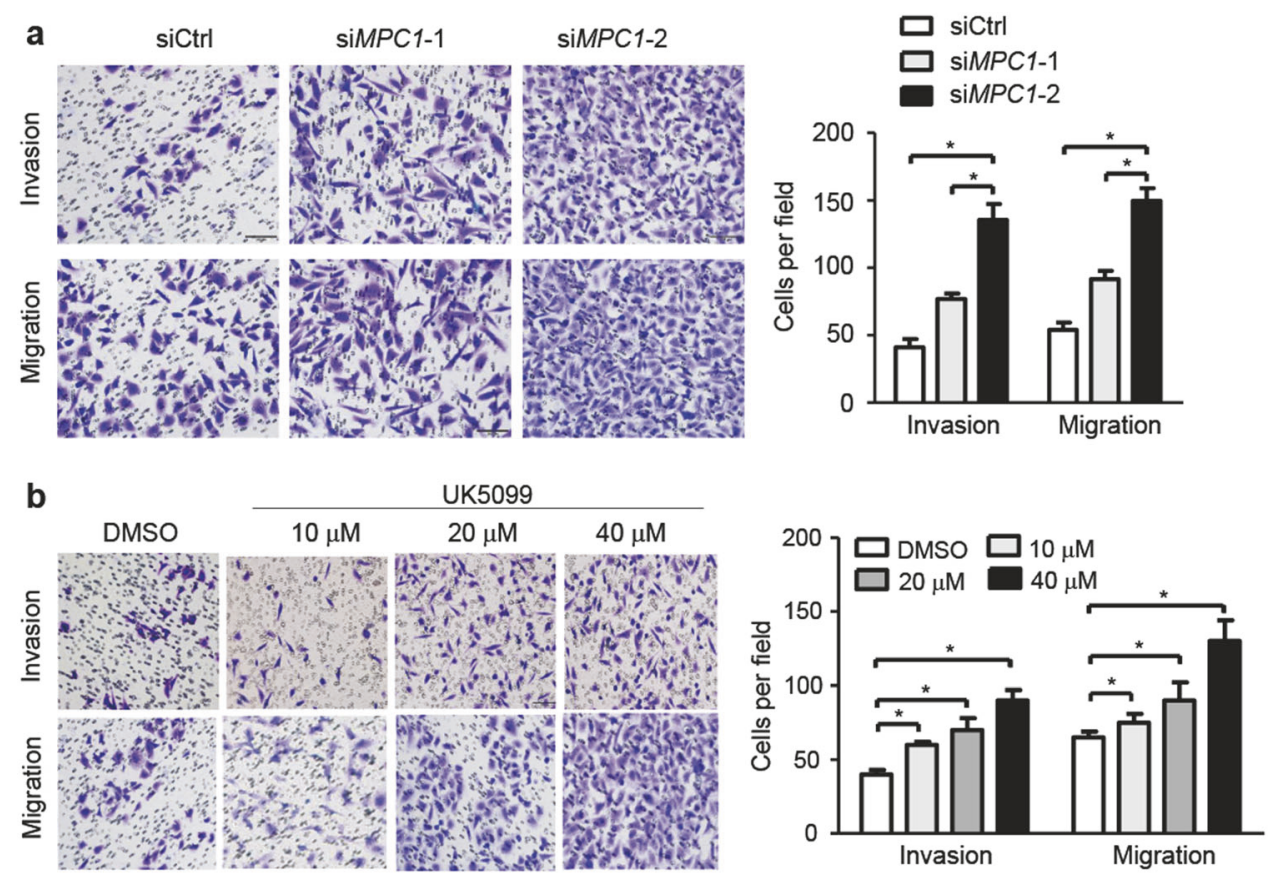

C

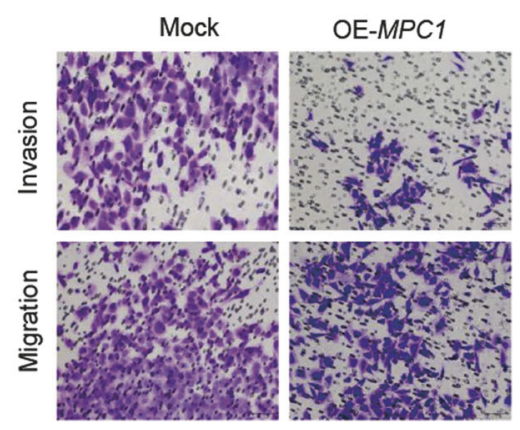

All significant statistical differences were defined as $* P<0.05, * * P<0.01$, and $* * * P<0.001$.

\section{Results}

\section{MPC1 is downregulated in RCC tissues}

We measured the expression of mitochondrial marker genes ( $n=40)$ in RCC and normal tissues including MPC1, cytochrome C (CYCS), TOMM6, and TOMM7 (Supplementary Figure 1a). MPC1, CYCS, TOMM6, and TOMM7 were examined in 10 pairs of $\mathrm{RCC}$ and corresponding adajacent non-cancerous tissue by qPCR. The results showed decreased MPC1 mRNA in RCC tissues compared with adjacent non-cancerous tissues (Supplementary Figure 1b). MPC1 and MPC2 proteins consisting of MPC were examined in 12 pairs of RCC and corresponding adjacent non-cancerous tissue by Western blotting, in which the expression of MPC1 was downregulated in RCC tissues (Fig. 1a, b). However, there was no difference in MPC2 expression in RCC versus adjacent non-cancerous tissue (Fig. 1a, b). Analysis of GEO database (GDS505) showed that the expression of MPC1 mRNA, but not MPC2 mRNA, was decreased in RCC tissues in comparison with normal kidney specimens (Fig. 1c). We also performed IHC using a 150-case RCC cohort (Cohort-150). In 30 paired cases from the Cohort-150, MPC1 expression was lower in tumor tissues than in corresponding adjacent non-cancerous tissue (Fig. 1d and e). In addition, the proportion of MPC $1^{\text {low }}$ cells was markedly higher in RCC tissues $(60.67 \%, 91 / 150)$ than that in corresponding adjacent non-cancerous tissue (33.33\%, 10/30) (Supplementary Table 3). Therefore, our data show downregulation of MCP1 expression in RCC versus corresponding adjacent non-cancerous tissue.

\section{MPC1 expression levels are correlated with the prognosis of RCC patients}

We next analyzed MPC1 expression and the clinicopathological features of RCC tissues from Cohort-150. Among these samples, 59 cases were defined as $\mathrm{MPC}^{\text {high }}$ 


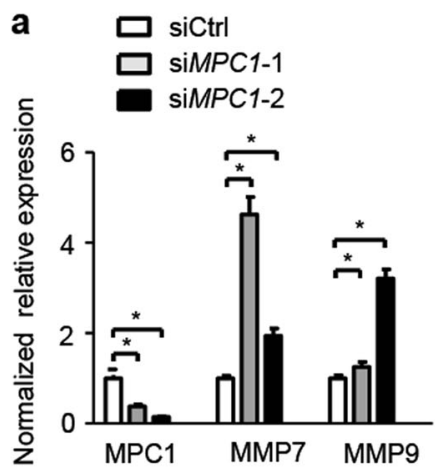

b
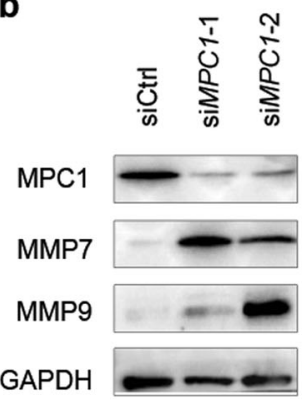

d
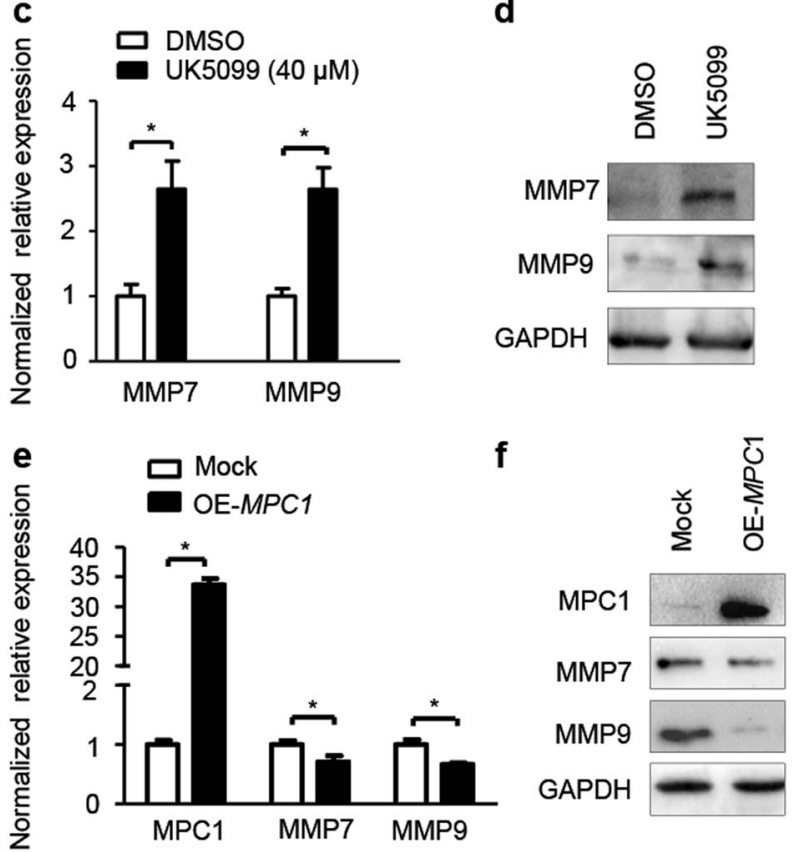

Fig. 4 Involvement of MPC1 in the migration and invasion of RCC cells through MMPs pathway. a The mRNA levels of MPC1, MMP7, and MMP9 in 786-O cells transfected with siCtrl and siMPC1 detected by qPCR. b MPC1, MMP7, and MMP9 proteins in 786-O cells transfected with siCtrl and siMPCl detected by Western blotting. GAPDH serves as a loading control. $\mathbf{c}$ qPCR analysis of mRNA levels of MMP7 and MMP9 in 786-O cells treated with UK5099, $* P<0.05$. d The expression of MMP7 and MMP9 in 786-O cells treated with UK5099 was analyzed by Western blotting. GAPDH serves as a loading control. e The mRNA levels of MPC1, MMP7, and MMP9 in 786-O cells transfected with Mock or $M P C 1(\mathrm{OE}-M P C 1), * P<0.05$. f The expression of MPC1, MMP7, and MMP9 in 786-O cells transfected with Mock or MPC1 (OE-MPC1) detected by Western blotting. GAPDH serves as a loading control

and $91 \mathrm{MPC} 1^{\text {low }}$. MPC1 expression was negatively correlated with tumor size $(\mathrm{T})(P=0.01)$ and TNM stages $(P=$ 0.01 ), but not with tumor location, histological grade, and patient gender (Supplementary Table 4). We also noticed that the expression of MPC1 protein in stage I RCC was significantly lower than that in higher stage RCC (stages II and III) (Fig. 2a, b). These results suggested that decreased expression of MPC1 in RCC was associated with tumor progression. Univariate and multivariate analyses further indicated that low MPC1 expression was an independent prognostic indicator for poor OS of RCC patients (Supplementary Table 5). Kaplan-Meier survival analysis using Cohort-150 showed significantly shorter OS for patients with MPC1 $1^{\text {low }} \mathrm{RCC}$ than those with MPC $1^{\text {high }} \mathrm{RCC}(P=$ 0.0016) (Fig. 2c). Moreover, survival analysis of 164 RCC patients from the TCGA-database consistently indicated that low of MPC1 expression was associated with poorer OS $(P=0.0128)$ (Fig. 2d). Thus, lower expression of MPC1 acts as an indicator of unfavorable prognosis for RCC patients.

\section{Loss of MPC1 promotes the migration and invasion of RCC cells in vitro}

Based on the function of MPC1 in the migration and invasion capacity of cholangiocarcinoma cells [9], we tested the effect of MPC1 on the mobility of RCC cells. We established MPC1-depletion and MPC1-overexpression subpopulations in 786-O cells (Supplementary Figure 2). Transwell assay with or without Matrigel showed that loss of MPC1 increased the migration and invasion capacity of RCC cells (Fig. 3a). Treatment with different concentrations of the MPC inhibitor [13], UK5099, enhanced RCC migration and invasion compared with vehicle-treated cells (Fig. 3b). In contrast, overexpression of MPC1 significantly impaired the migration and invasion of RCC cells in vitro (Fig. 3c). These results suggested that loss of MPC1 was associated with increased mobility and invasiveness of RCC cells.

\section{Matrix metalloprotein, but not EMT, is affected by MPC1 in RCC cells}

To define the downstream targets of MPC1 linked to RCC cell migration and invasion, we examined two EMT markers, vimentin and E-cadherin. Neither of the two genes was changed in their levels after MPC1 depletion, MPC inhibitor treatment, or MPC1 overexpression (Supplementary Figure 3a-c). Since matrix metalloproteinases (MMPs) play pivotal roles in cancer cell migration and invasion [14], we then evaluated the expression of MMPs. The mRNA and protein levels of MMP7 and MMP9 were significantly upregulated in 786-O cells transfected with siMPC 1 versus siCtrl constructs (Fig. 4a, b). Treatment of RCC cells with the MPC inhibitor, UK5099 also increased the expression of MMP7 and MMP9 (Fig. 4c, d). However, RCC cells overexpressing MPC1 showed reduction in the expres- 
Fig. 5 Involvement of MPC1 in the migration and invasion of RCC cells through regulation of HIF1 $\alpha$. a The expression of MPC1 in 786-O cells treated with $200 \mu \mathrm{M} \mathrm{CoCl}{ }_{2}$ in normal $\left(20 \% \mathrm{O}_{2}\right)$ or hypoxia conditions $\left(1 \% \mathrm{O}_{2}\right)$. b Western blotting of MPC1 and HIF1 $\alpha$ in 786-O cells transfected with siCtrl and siMPC1. GAPDH serves as a loading control. c The expression of HIF1 $\alpha$ in Mock and $\mathrm{OE}-\mathrm{MPCl}$ cells detected by Western blotting. GAPDH serves as a control. d Representative IHC staining for MPC 1 and HIF $1 \alpha$ in RCC tissues. Black arrows: cancer cells. e $\chi^{2}$ analysis of the percentage of HIF $1 \alpha^{\text {high }}$ and $\mathrm{HIF} 1 \alpha^{\text {low }}$ cells in MPC1 $1^{\text {low }}$ and MPC $1^{\text {high }} \mathrm{RCC}$ specimens $(n=150)$

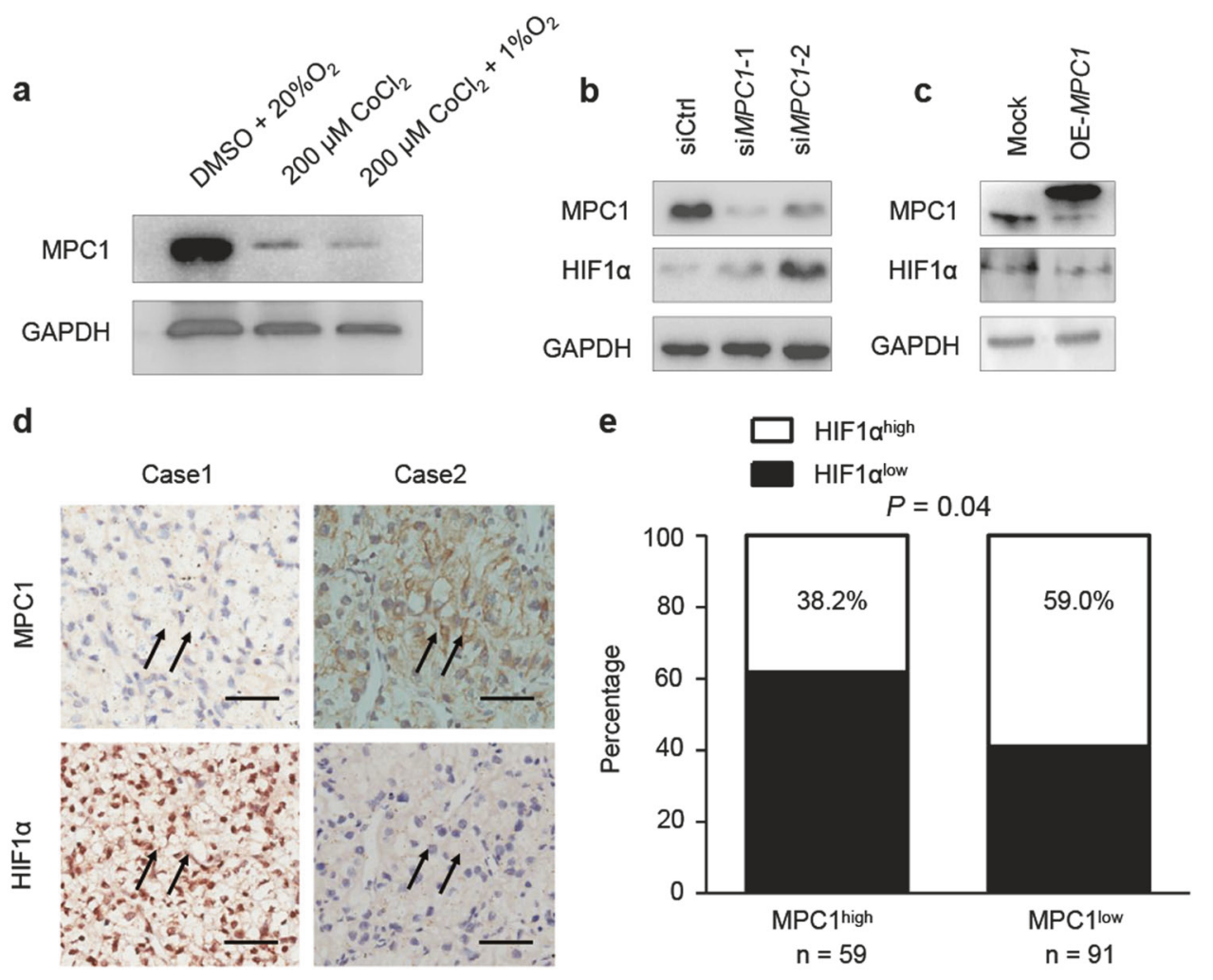

sion of MMP7 and MMP9 (Fig. 4e, f). Thus, loss of MPC1 enhances the mobility and invasiveness of RCC cells through upregulation of MMPs.

\section{MPC1 is involved in the pathway of hypoxia/HIF1a axis in RCC cells}

Hypoxia frequently occurs in RCC [15] that leads to increased cell migration and invasion [16, 17]. In addition, proteins in mitochondria are highly sensitive to regulation by hypoxia $[18,19]$. We therefore speculated that MPC1 might be regulated by hypoxia. $\mathrm{CoCl}_{2}$ treatment or culture under $1 \% \mathrm{O}_{2}$ to mimic hypoxia [20], reduced the expression of MPC1 in RCC (Fig. 5a and Supplementary Figure $4 a)$. Since HIF1 $\alpha$ is a crucial regulator of hypoxia [21] and is linked to the progression of RCC [22], we investigated the relationship between MPC1 and HIF1 $1 \alpha$. We observed that suppression of MPC1 expression increased HIF1 $\alpha$ expression in RCC (Fig. 5b and Supplementary Figure 4b). In contrast, overexpression of MPC1 decreased HIF1 $\alpha$ (Fig. 5c). Moreover, in specimens from Cohort-150, we found that the expression levels of HIF1 $\alpha$ in MPC $1^{\text {low }}$ RCC cells were higher than that in MPC $1^{\text {high }}$ RCC cells (Fig. $5 \mathrm{~d}$, e), a finding also confirmed using TCGA database (Supplementary Figure 4c). These results suggest the possibility of MPC1 as an upstream regulator of HIF $1 \alpha$ in RCC cells.

\section{Overexpression of MPC1 reduces the growth of RCC xenograft tumor}

To examine the effect of MPC1 on the malignant behavior of RCC cells in vivo, RCC cells (786-O) with overexpressing or knockdown MPC1 were subcutaneously implanted into immunocompromised mice (Fig. 6a, b). The average weight and volume of tumors formed by RCC cells with MPC1 knockdown grew more rapidly than those formed by RCC cells overexpressing MPC1 (Fig. 6c, d). IHC staining of xenograft tumors showed that the expression of MMP7 and HIF1 $\alpha$ was decreased in tumors derived from MPC1 overexpressing RCC cells as compared with those derived from Mock-transfected cells (Fig. 6e). Therefore, xenograft data demonstrate that higher level of MPC1 expression is associated with decreased rate of the growth of RCC and the expression of MMP7 and HIF1 $\alpha$ in tumors.

\section{Discussion}

RCC contains metabolic alterations characterized by increased glycolysis rate and lactate production under normoxia condition known as Warburg effect [23], which is correlated with pyruvate metabolism and attenuation of pyruvate transportion [24]. Therefore, pyruvate- 
a

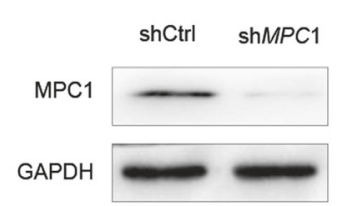

C

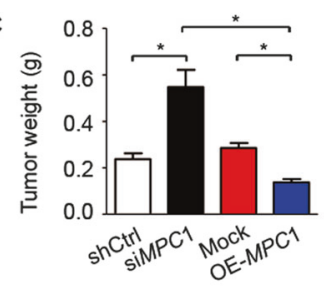

e
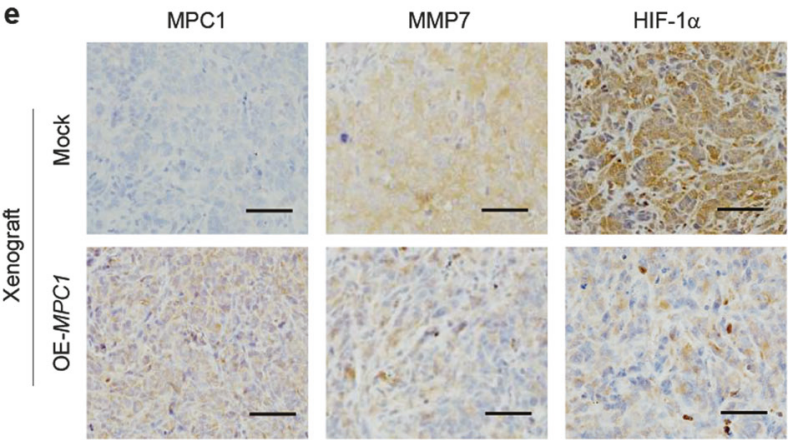

Fig. 6 The tumorigenesis of RCC cells transfected with MPC1 in mice. a Western blotting of MPC1 in Scramble (shCtrl) and shMPC1 transfected 786-O cells. b Subcutaneous tumor formation on day 30 in NOD-SCID mice by Mock, OE-MPC1, shCtrl, and shMPC1 786-O cells $\left(1 \times 10^{6}\right.$ cells/mice $)$. $\mathbf{c}$ and $\mathbf{d}$ The weight $(\mathbf{c})$ and volume $(\mathbf{d})$ of tumors formed by Mock, OE-MPC1, shCtrl, and shMPC1 786-O cells in mice, $* * P<0.01 ; * P<0.05$. e Representative IHC staining for MPC1, MMP7, and HIF1 $\alpha$ in tumors formed by Mock and OE-MPC1 786-O cells. Scale bar $=20 \mu \mathrm{m}$

transporting-related proteins may play critical roles during tumorigenesis and tumor progression. In this study, we report that MPC1 was significantly downregulated in RCC tissues, with more advanced stages and poorer OS of patients. In vitro, the migration and invasion of RCC cells were enhanced when MPC1 was depleted with siRNA or suppressed by MPC inhibitor. Thus, MPC1 acts as a tumor suppressor in RCC.

It has been reported that MPC1 expression is reduced in various types of cancer [25-27]. Deficiency of MPC1 contributes to the dysregulation of mitochondria-related metabolism that leads to oncogenic switch of metabolic profiles [5, 27]. In cancer cells, MPC1 deficiency or mutation-mediated inactivation promote aerobic glycolysis and stemness [28]. Although both MPC1 and MPC2 are simultaneously downregulated in some types of cancer [57], we observed that only MPC1 was aberrantly regulated in RCC. MPC1 and MPC2 form a multimeric complex to control pyruvate transportation [4, 29], and both are required for complex stability $[5,30]$. Therefore, either MPC1 or MPC2 aberrant regulation may result in functional loss to promote tumorigenesis. It has been found that suppression of MPC1 is sufficient to interfere with mitochondrial pyruvate uptake and oxidation critically involved in Warburg effect in cancer cells [5].

Low expression of MPC1 in cancer cells leads to increased cell glycolysis which is responsible for enhanced invasive phenotype $[6,31]$. Hypoxia is also tightly associated with glycolysis, and plays pivotal roles in promoting tumor progression, including RCC $[15,32]$. We observed that hypoxia in RCC cells resulted in MPC1 downregulation and MPC1 depletion increased HIF1 $\alpha$ expression. Moreover, MMPs, but not EMT, was upregulated by MPC1 deficiency, which may be responsible for increased invasiveness of RCC cells.

In conclusion, loss of MPC 1 expression in RCC predicts poor survival of patients. MPC1 reduces the mobility and invasiveness of RCC cells by inhibiting HIF1 $\alpha$ and MMPs expression. Therefore, MPC1 plays a tumor-suppressor function in RCC and acts as a prognostic indicator.

Acknowledgements This project was supported by grants from the National Natural Science Foundation of China (Nos. 81372684 and 81230062 ).

\section{Compliance with ethical standards}

Conflict of interest The authors declare that they have no conflict of interest.

\section{References}

1. Levi F, Ferlay J, Galeone C, et al. The changing pattern of kidney cancer incidence and mortality in Europe. BJU Int. 2008;101:949-58.

2. Tan AS, Baty JW, Dong LF, et al. Mitochondrial genome acquisition restores respiratory function and tumorigenic potential of cancer cells without mitochondrial DNA. Cell Metab. 2015;21:81-94.

3. Camarda R, Zhou AY, Kohnz RA. Inhibition of fatty acid oxidation as a therapy for MYC-overexpressing triple-negative breast cancer. Nat Med. 2016;22:427-32.

4. Herzig S, Raemy E, Montessuit S, et al. Identification and functional expression of the mitochondrial pyruvate carrier. Science. 2012;337:93-96.

5. Schell JC, Olson KA, Jiang L, et al. A role for the mitochondrial pyruvate carrier as a repressor of the Warburg effect and colon cancer cell growth. Mol Cell. 2014;56:400-13.

6. Wang L, Xu M, Qin J, et al. MPC1, a key gene in cancer metabolism, is regulated by COUPTFII in human prostate cancer. Oncotarget. 2016;7:14673-83.

7. Li X, Ji Y, Han G, et al. MPC1 and MPC2 expressions are associated with favorable clinical outcomes in prostate cancer. BMC Cancer. 2016;16:894.

8. Li Y, Li X, Kan Q, et al. Mitochondrial pyruvate carrier function is negatively linked to Warburg phenotype in vitro and malignant features in esophageal squamous cell carcinomas. Oncotarget. 2017;8:1058-73. 
9. Ohashi T, Eguchi H, Kawamoto K, et al. Mitochondrial pyruvate carrier modulates the epithelial-mesenchymal transition in cholangiocarcinoma. Oncol Rep. 2018;39:1276-82.

10. Wu HB, Yang S, Weng HY, et al. Autophagy-induced KDR/ VEGFR-2 activation promotes the formation of vasculogenic mimicry by glioma stem cells. Autophagy. 2017;13:1528-42.

11. Flores A, Schell J, Krall AS, et al. Lactate dehydrogenase activity drives hair follicle stem cell activation. Nat Cell Biol. 2017;19:1017-26.

12. Camp RL, Dolled-Filhart M, Rimm DL. X-tile: a new bioinformatics tool for biomarker assessment and outcome-based cutpoint optimization. Clin Cancer Res. 2004;10:7252-9.

13. Schell JC, Wisidagama DR, Bensard C, et al. Control of intestinal stem cell function and proliferation by mitochondrial pyruvate metabolism. Nat Cell Biol. 2017;19:1027-36.

14. Mikami S, Oya M, Mizuno R, et al. Recent advances in renal cell carcinoma from a pathological point of view. Pathol Int. 2016;66:481-90.

15. Mehdi A, Riazalhosseini Y. Epigenome aberrations: emerging driving factors of the clear cell renal cell carcinoma. Int J Mol Sci. 2017; 18:E1774.

16. Ma X, Gu L, Li H, et al. Hypoxia-induced overexpression of stanniocalcin-1 is associated with the metastasis of early stage clear cell renal cell carcinoma. J Transl Med. 2015;13:56.

17. Koh MY, Nguyen V, Lemos R Jr, et al. Hypoxia-induced SUMOylation of E3 ligase HAF determines specific activation of HIF2 in clear-cell renal cell carcinoma. Cancer Res. 2015;75:316-29.

18. Li P, Zhang D, Shen L, et al. Redox homeostasis protects mitochondria through accelerating ROS conversion to enhance hypoxia resistance in cancer cells. Sci Rep. 2016;6:22831.

19. Bell EL, Klimova T, Chandel NS. Targeting the mitochondria for cancer therapy: regulation of hypoxia-inducible factor by mitochondria. Antioxid Redox Signal. 2008;10:635-40.
20. Shweta, Mishra KP, Chanda S, et al. A comparative immunological analysis of $\mathrm{CoCl} 2$ treated cells with in vitro hypoxic exposure. Biometals. 2015;28:175-85.

21. Guan SY, Leng RX, Tao JH, et al. Hypoxia-inducible factor1alpha: a promising therapeutic target for autoimmune diseases. Expert Opin Ther Targets. 2017;21:715-23.

22. Gudas LJ, Fu L, Minton DR, et al. The role of HIF1alpha in renal cell carcinoma tumorigenesis. J Mol Med. 2014;92:825-36.

23. Warburg O. On the origin of cancer cells. Science. 1956;123:309-14.

24. Eboli ML, Paradies G, Galeotti T, et al. Pyruvate transport in tumour-cell mitochondria. Biochim Biophys Acta. 1977;460:183-7.

25. Hiller K, Metallo CM. Profiling metabolic networks to study cancer metabolism. Curr Opin Biotechnol. 2013;24:60-68.

26. Yang C, Ko B, Hensley CT, et al. Glutamine oxidation maintains the TCA cycle and cell survival during impaired mitochondrial pyruvate transport. Mol Cell. 2014;56:414-24.

27. Compan V, Pierredon S, Vanderperre B, et al. Monitoring mitochondrial pyruvate carrier activity in real time using a BRETbased biosensor: investigation of the Warburg effect. Mol Cell. 2015;59:491-501.

28. Li X, Han G, Li X, et al. Mitochondrial pyruvate carrier function determines cell stemness and metabolic reprogramming in cancer cells. Oncotarget. 2017;8:46363-80.

29. Bricker DK, Taylor EB, Schell JC, et al. A mitochondrial pyruvate carrier required for pyruvate uptake in yeast, Drosophila, and humans. Science. 2012;337:96-100.

30. Bender T, Pena G, Martinou JC. Regulation of mitochondrial pyruvate uptake by alternative pyruvate carrier complexes. EMBO J. 2015;34:911-24.

31. Gu Y, Song Y, Liu Y. [Clinical characteristics and prognostic factors of pulmonary tuberculosis with concurrent lung cancer]. Zhonghua Yi Xue Za Zhi. 2014;94:2838-40.

32. Harris AL. Hypoxia-a key regulatory factor in tumour growth. Nat Rev Cancer. 2002;2:38-47. 